\title{
Zu Lydus de ostentis.
}

Der Codex Parisinus supplementi graeci 20 ist eine Sammelhandschrift des 17. Jahrhunderts und enthält auf 296 Papierfoliis astronomische Exzerpte, die sich Ismaël Bouillaud und andere Gelehrte seiner Zeit aus älteren Handschriften der damaligen Bibliotheca regia gemacht haben. Ein solches Exzerpt, das aus dem Codex regius 1113 stammt dessen Identität ich leider nicht feststellen konnte, da die Handschriften jetzt einer anderen Zählung folgen - lautet (f. 208'):

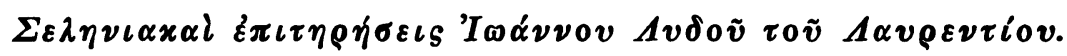

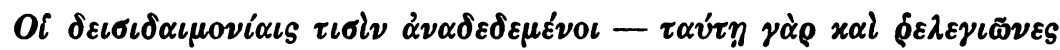

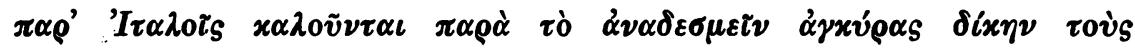

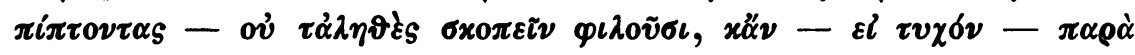

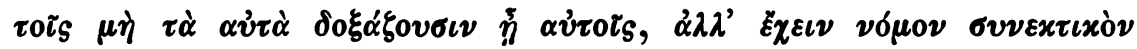

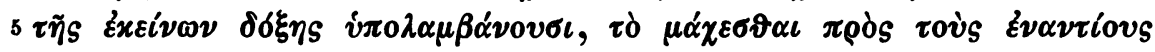

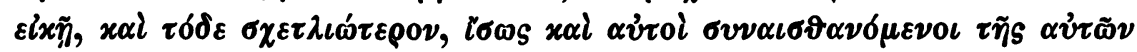

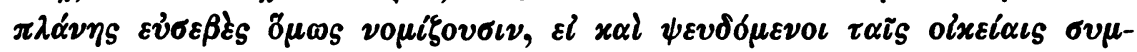

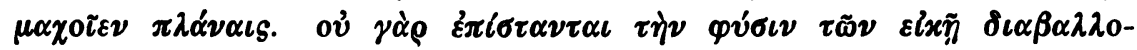

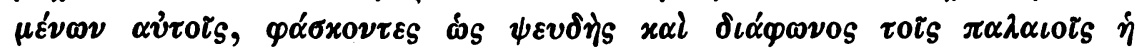

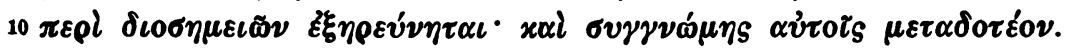

Wegen der künstlichen Wortstellung und Satzkonstruktion dieses Abschnittes möge zunächst eine Übersetzung folgen, die den Sinn, wie ich ihn zu verstehen glaube, wiedergiebt:

Quicumque religione aliqua religati sunt - eam enim ob rem etiam appellatur religio apud Italos a religando labentes tamquam ancora non verum solent respicere, etsi - si casu fit - apud eos est, qui non idem probant, quod ipsi: sed legem opiniones illorum complectentem sese habere respondent, repugnare adversariis ratione nulla habita; atque hoc etiam atrocius: licet et ipsi erroris sui conscii fiant, pium tamen ducunt, auxilio venire proprio suorum errori quamvis mentientes.

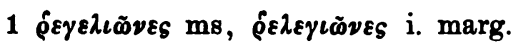

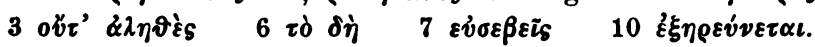


neque enim rerum naturae scientiam eorum, quos temere ipsi calumniantur, noscunt, dum commemorant, quam falso et inconcinne investigata sit a veteribus illa de ostentis (scientia): et ignoscendum esse illis.

Eine genauere Betrachtung dieses Exzerptes zeigt uns, dafs kein Grund vorhanden ist, an der Herkunft unseres Stückes aus Lydus zu zweifeln: sowohl die Form - man beachte namentlich im Eingange die lateinische Etymologie - als auch der Inhalt - zum Vergleiche

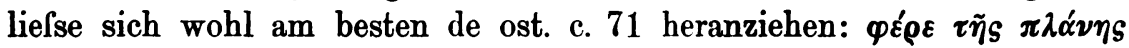

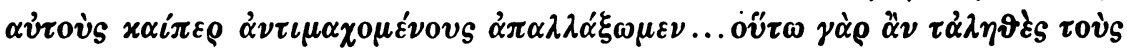

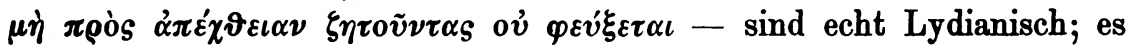
fragt sich nur, welchem der erhaltenen Werke des Lydus wir unseren Passus einzuordnen haben. Dazu giebt uns der Z. 10 stehende Ausdruck $\delta \iota \sigma \eta \mu \varepsilon i \alpha$ einen Fingerzeig, und die Überschrift $\sigma \varepsilon \lambda \eta \nu \iota \alpha x \alpha i l z \pi l-$ $\tau \eta \varrho \eta^{\prime} \varepsilon \iota s$ scheint uns auf c. 42 und folgende des Buches de ostentis zu

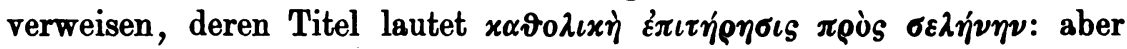
hier fehlt nichts, und in dem Vorhandenen ist keine Spur zu finden, dafs etwas dem Pariser Exzerpte Ähnliches dort gestanden haben könnte; wir müssen daher annehmen, dafs der Titel mit einem Zitat aus einem

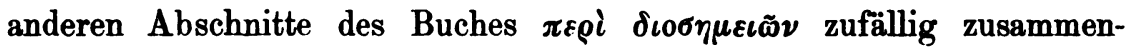

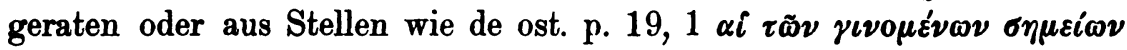
$\pi \varepsilon \rho i \ldots \tau \dot{\eta} \nu \sigma \varepsilon \lambda \dot{\nu} \nu \eta \nu \pi \alpha \rho \alpha \eta \emptyset \eta \dot{\sigma} \sigma \varepsilon \iota_{S}$ neu gebildet ist, und es gilt, den Zusammenhang $\mathrm{zu}$ finden, in den unsere Zeilen hineinpassen. Lydus setzt sich in denselben mit den Orthodoxen auseinander, die über die Physik der Alten, insbesondere deren Theorie von den Himmelserscheinungen, absprechend urteilen, ohne sie hinreichend zu kennen. Sehen wir uns nun den Anfang des Buches $\pi \varepsilon \rho i ~ \delta \iota 0 \sigma \eta \mu \varepsilon i \tilde{\nu} \nu$ an. Es sind - nach Hases Urteil - die drei bis vier ersten Zeilen verloren

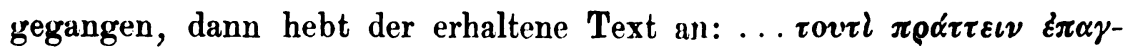

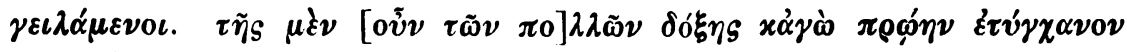

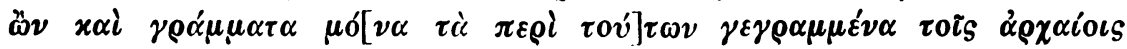

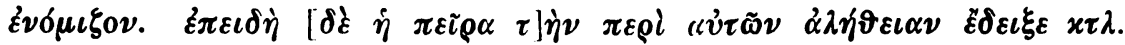
Man sieht, Lydus hatte in dem verlorenen Stücke von Leuten gesprochen, die die Kunde der Alten von den Himmelserscheinungen gering schätzten. Man wird auch aus dem Erhaltenen schliefsen können, dafs sich $\dot{\varepsilon} \pi \alpha \gamma \gamma \varepsilon \iota \lambda \alpha$ ́́ $\mu \varepsilon \nu o \iota$ auf eine gebildete Minderzahl bezieht, die ihre

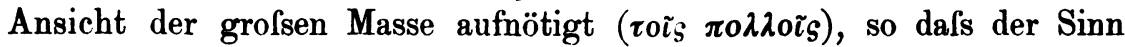
vor $\tau o v \tau i$ die Ergänzung eines ähnlichen Dativs, etwa von $\pi \tilde{\alpha} \sigma \iota$, fordert. Im Vorausgegangenen mufs dann die Ansicht dieser Minderzahl auseinandergesetzt worden sein, und zwar so, dafs das letzte von ihr ausgesagte Zeitwort nicht sowohl ein Wort des Denkens, als des Handelns 


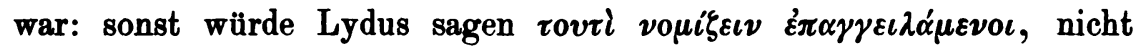

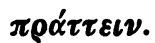

Sehen wir nun nach diesen Bemerkungen noch einmal das Pariser Exzerpt an, so springt in die Augen, dals es die Anforderungen, die an den verlorenen Anfang des Buches de ostentis gestellt werden, vollständig erfüllt; auch macht seine Verbindung mit dem erhaltenen Text keine Schwierigkeit, sobald man liest:

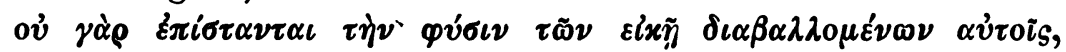

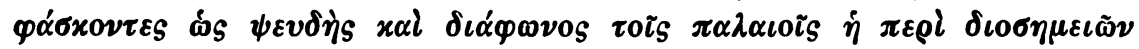

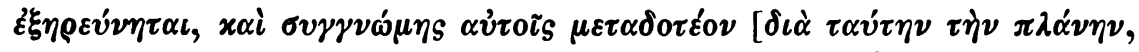

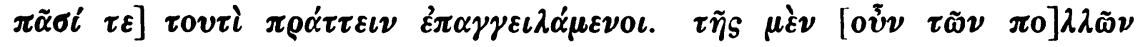

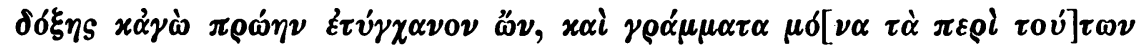

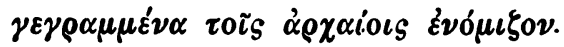

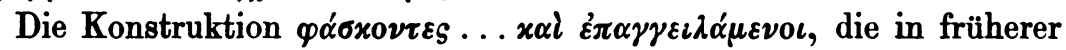
Zeit anstöIsig wäre, hat für Lydus nichts Auffallendes mehr; auf diese Weise wäre dann der Anfang des Buches de ostentis hergestellt. Dafs die Anlage des Werkes in seinem Fortgange eine andere war, als die heute erhaltene, zeigt der Schlufs des Werks de mensibus, IV c. 97:

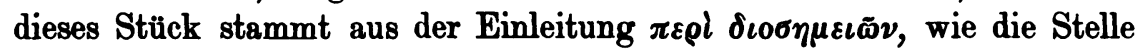

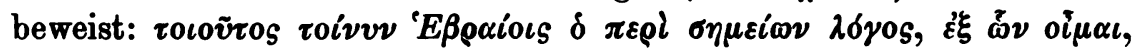

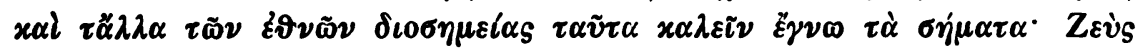

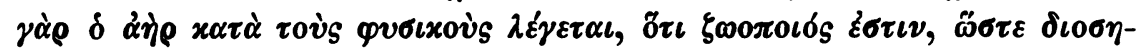

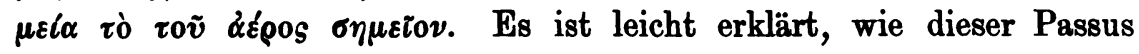
an den Schlufs von de mensibus geriet: der Ausschreiber hatte in seinem Eifer noch in das folgende Werk hineinexzerpiert. Dafs die Reihenfolge de mensibus ostentis magistratibus war, beweist der Caseolinus (Wachsmuth de ostentis p. IX). Ob man aus demselben Grunde die Stelle über den Blitz, das letzte Exzerpt der Planudeischen, das drittletzte der Barberinischen Redaktion (de mens. III, $52=$ IV, 96), gleichfalls dem Buche de ostentis zusprechen darf, wage ich zur Zeit, wo mir das Material zu $\pi \varepsilon \rho l ~ \mu \eta \nu \tilde{\nu} \nu$ noch nicht in seiner Gesamtheit vorliegt, nicht zu entscheiden. Zwischen zwei Abschnitten aus de ostentis, d. h. ziemlich freien Umschriften, die für den eigentlichen

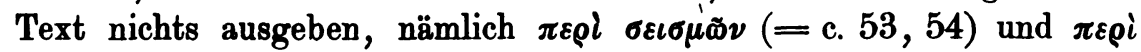

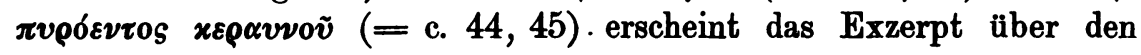
Blitz ( $\pi \varepsilon \rho l \sigma x \eta \pi \tau \tilde{\omega} \nu$ ) im cod. Paris. suppl. gr. $607 \mathrm{~A} \mathrm{f.} 69 \mathrm{v}$ (Treu, Ohlauer Progr. 1880 p. 46), aber die Anordnung scheint ursprünglich

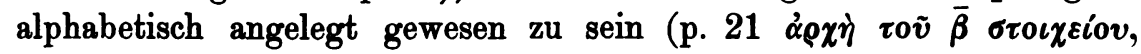

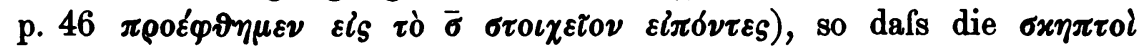
sehr wohl nach den $\sigma \varepsilon \iota \sigma \mu o l$ folgen konnten, ohne mit ihnen aus demselben Buche zu stammen. Jedenfalls darf man gegenwärtig auf diese 
Stelle aufmerksam machen, da sie an Interesse gewonnen hat durch die Anecdota medica Graeca von Fuchs (Rh. M. L, 576 sqq.); sie korrigiert auch die sinnlosen Worte des Theophanes Nonnus (p. 584):

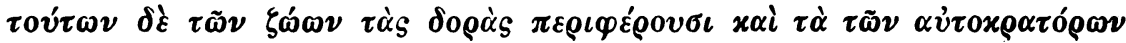

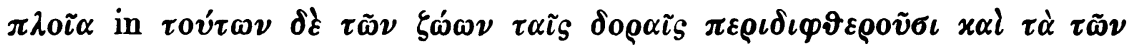
$\left.\alpha \dot{v} \tau 0 x \rho \alpha \tau \dot{\rho} \rho \omega \nu \pi \lambda 0 i \alpha^{1}{ }^{1}\right)$

Sodann sei es gestattet, einiges Handschriftliche zu dem Buche

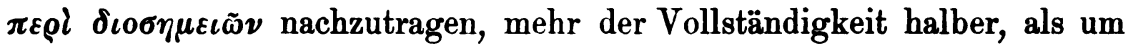
neue Resultate beizubringen. In seiner Vorrede zu der Ausgabe von de ostentis erwähnt Wachsmuth p. XVI, in dem cod. Venetus 234 finde sich f. $284^{\mathrm{v}}-287^{\mathrm{v}}$ des Nigidius Traktat über den Donner (c. $27-38$ ) und f. $287^{\vee}-288^{\vee}$ des Vicellius Abschnitt über das Erdbeben (c. 54 Mitte-58), beide noch nicht benutzt. Gemeint ist, wie auch S. X und 2 richtig steht, der cod. Marcianus 324, eine astronomische Sammelhandschrift des 14. Jahrhunderts, eingesehen von Hase, der auf dem Vorsatzblatt vermerkt hat C. B. Hase Germanus. Er ent-

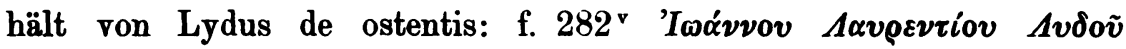

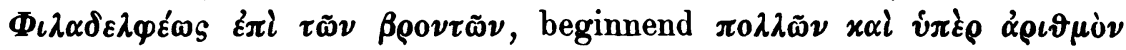

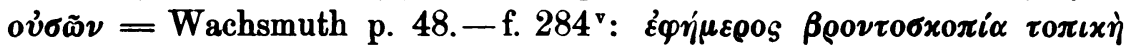

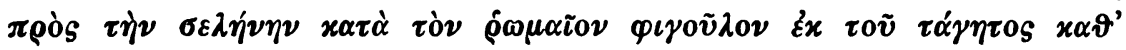

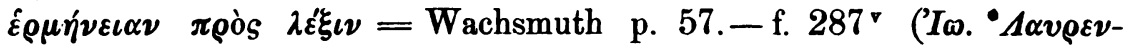

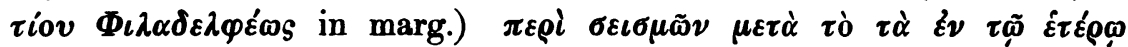

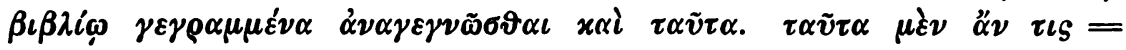
Wachsmuth p. 105; der Zusatz $\mu \varepsilon \tau \grave{\alpha}$ iò $x \tau \lambda$. wiederholt sich im Vat. Pal. 312. Neues ergiebt die Handschrift nicht. - Ein anderer Codex Venetus bibliothecae S. Marci (II 15), die Werke des Photius von der Hand des Antonius Catiforus Zacynthius (s. XVIII) enthaltend, weist

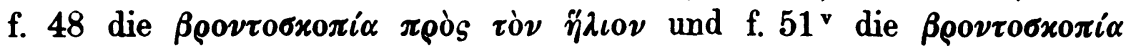
$\pi \rho o ̀ s ~ \sigma \varepsilon \lambda \eta^{\prime} \nu \eta \nu$ auf, bemerkt aber zugleich dabei, dafs sie e codice Palatino num. 312 genommen seien: in diesem war $\Phi$ $\tau$ íiog verschrieben (Wachsmuth $\mathrm{p}$. XIV).

Der Haseschen Ausgabe folgend, erwähnt Wachsmuth ferner das Kalendarium des Clodius Tuscus in dem codex Barberinus 177, aus dem die Handschriften des Marini und des Bigot (jetzt Par. gr. 3084) abgeschrieben seien. Es ist der cod. Barb. II 15 (num. 277), eine Papierhandschrift des 15. Jahrhunderts, die aufser den Exzerpten de mensibus enthält die 'Ephemeris sive Diarium sive Kalendarium ex Claudio Thusco', wozu eine spätere Hand bemerkt hat: 'Sex. Clodius fastos grece scripsit, ef. Lactant. de fatua I'. Was das Abhängigkeits-

1) Zur Sache selbst siehe Rohde, Psyche 363, 2. 466. 
verhältnis des Apographum Marinianum und Bigotianum vom Barberinus angeht, was schon bei de mensibus nicht ganz gesichert ist,

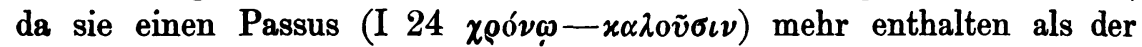
Barberinus, so kann von ihm gar nicht die Rede sein bei dem Kalendarium, wie wir gleich sehen werden. Zwar das Apographum Marinianum, das in Rom leider nicht aufzufinden war, scheidet aus, doch haben wir das Zeugnis des Parisinus.

Im Barberinus beginnt sofort nach dem letzten Exzerpte aus de mensibus:

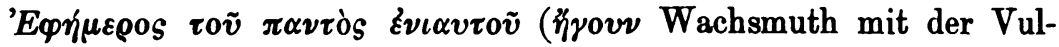

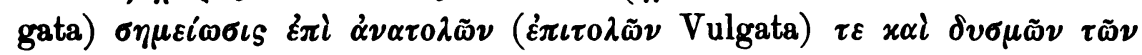

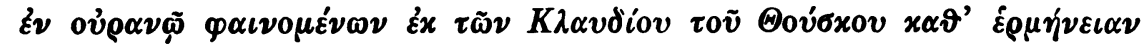
$\pi$ øòs $\lambda \varepsilon^{\prime} \xi \iota v$.

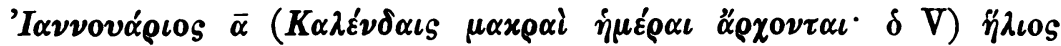

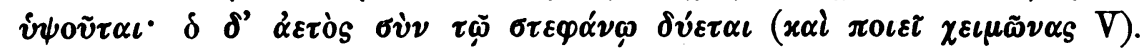

Die Handschrift enthält also, wie diese Probe zeigt, eine kürzere Fassung als die gewöhnliche. Das Kalendarium geht bis zum $\mu \eta \nu$ 'Iov́dıos.

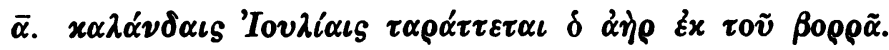

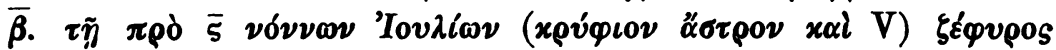

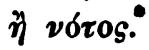

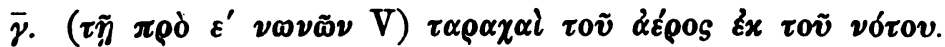

Damit bricht die Schrift ab, und zwar mitten auf der Seite. In dem Par. gr. 3084 dagegen, der keine wesentlichen Abweichungen von der Vulgata zeigt, geht das Kalendarium bis zum 5. Dezember. Es

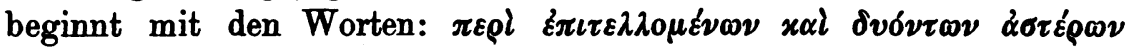

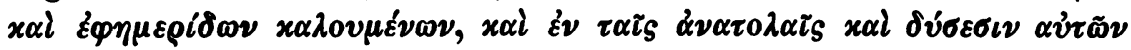

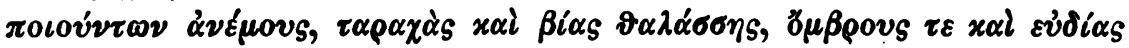

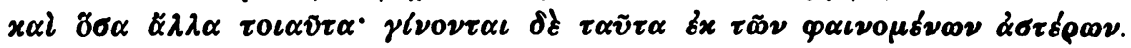

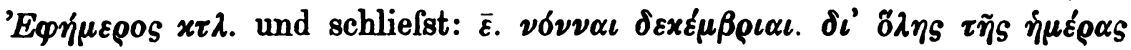

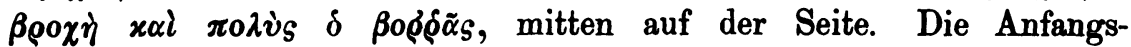
bemerkung hat unsere Handschrift mit dem Laur. 28, 34 gemein, der ebenso mit dem 5. Dezember abbricht ${ }^{1}$ ): mit demselben Tage schliefst auch der Paris. gr. 1991. Wir haben es also hier mit einer Handschriftenklasse zu thun, die vielleicht mit dem Archetypus des Barberinus verwandt ist, zu ihm selbst aber in einem gewissen Gegensatze steht.

Wenn Wachsmuth ferner an derselben Stelle (praef. p. XVI) be-

1) Die Madrider Hs scheint bis zum 23. Nov. zu gehen; siehe Martin, Missions scientifiques II 1892 p. $109 \mathrm{f}$. 
richtet, dafs sich Stücke aus c. 71 in dem Vat. gr. 1202 finden, so wäre vielleicht nachzutragen, dafs dieselben Exzerpte im Vat. gr. 1065 (bombyc. s. XIV) f. 21 ${ }^{\text {r }}$ stehen, und dafs 1065 der Archetypus von 1202 ist, aber eine bereits so getrübte Quelle, dafs wir aus ihr nichts für den ursprünglichen Lydustext schöpfen können. Diese vatikanischen Fragmente stehen übrigens mit geographischen Exzerpten zusammen, ohne dafs ein neuer Autor genannt wird, und so kommt es, dafs Creuzer (Meletemata p. 91) aus einem codex Leidensis, einm Verwandten des Vaticanus, fragmenta geographica des Lydus herausgab.

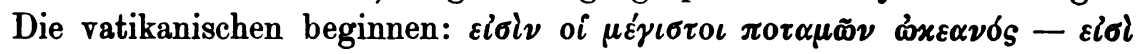

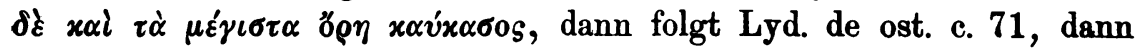

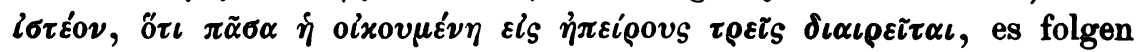
die Namen der Meere und Winde, die Grenzen der Erdteile, sodann

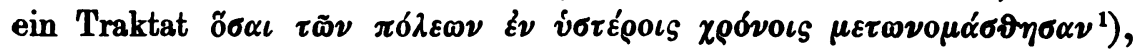
zu dem eine jüngere Hand im Par. gr. 1766 f. 410 - der ebenso wie der Vat. Pal. gr. 209 f. 285 denselben Traktat in etwas ausführlicherer Fassung enthält - zugeschrieben hat: Constantini de themat. p. 35. Im codex Caseolinus steht vor c. 71, Zeile 20 am Rande von der Hand

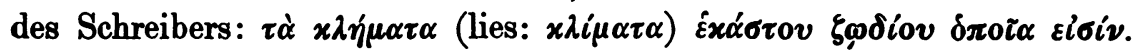

Zum Schlusse möchte ich noch zwei, wie ich glaube, unbekannte Traktate veröffentlichen, die gewissermalsen eine Ergänzung zu des Lydus Buch über die Himmelszeichen geben, indem sie zeigen, wie der Aberglaube, der jenen und seine Vorgänger beherrschte, im ganzen Mittelalter, verbreitet durch sein Werk oder unabhängig von demselben, bestanden hat. De ost. c. 27 sqq. enthalten die von Lydus übernommenen

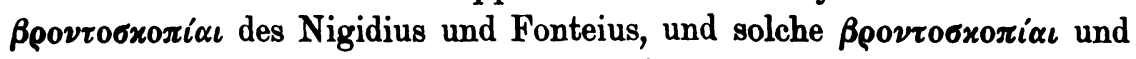

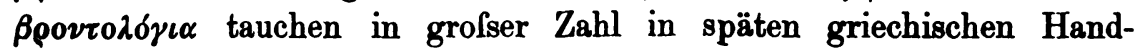
schriften auf $^{2}$ ), unter einander in der Ausdeutung verschieden, aber von derselben Anschauung ausgehend, dals der Donner an einem jeden Tage eine bestimmte andere Vorbedeutung habe. Meist sind diese Brontoskopien anonym; nur die des Par. gr. 2286 f. $109^{\vee}$ wird dem Zoroaster zugeschrieben. ${ }^{3}$ ) Als Muster ihrer Gattung möchte ich die einer Mailänder Handschrift wählen.

1) Verwandt mit den von Parthey, Hieroclis Synecdemus p. 311-818 abgedruckten Traktaten.

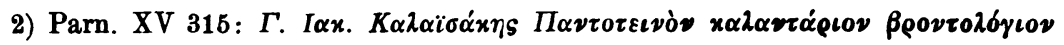

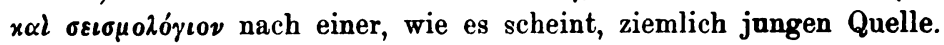

3) Wachsmuth de ost. XXXII: habent... aliquid momenti [quae in Lydo reperiuntur disputationes astrologicae], si Byzantinorum spectas tempora, quod primitiae sunt studiorum in eis mirum in modum excultorum et propagatorum. nam per tota Byzantinorum saecula superstitiosa summis in deliciis tales disqui- 
Cod. Ambr. E 81 sup. miscellaneus chart. s. XV, grammaticalia continens, f. 315r:

$$
\Pi \varepsilon \rho \curlywedge \beta \rho \circ \nu \tau \tilde{\omega} \nu .
$$

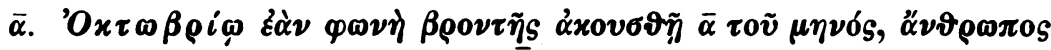

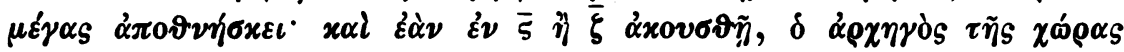

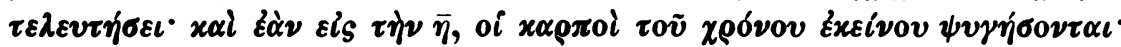

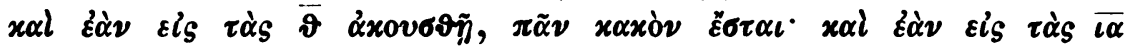

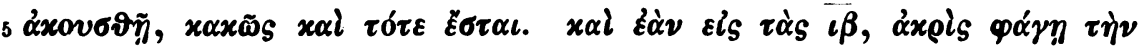

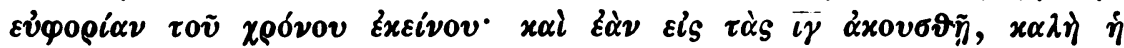

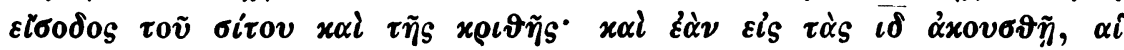

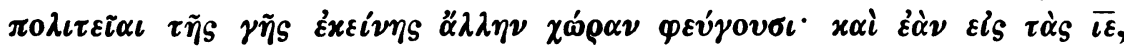

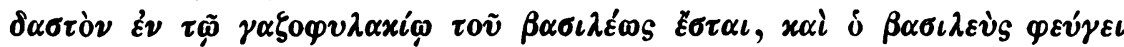

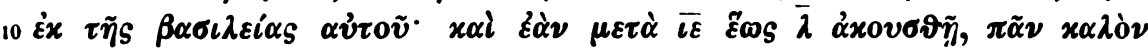

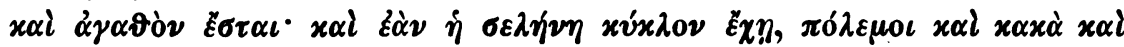

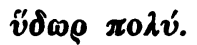

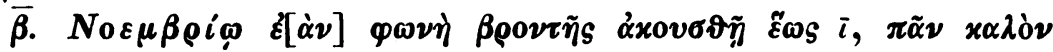

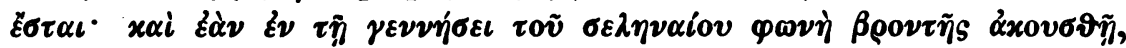

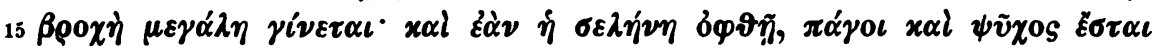

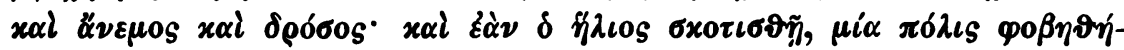

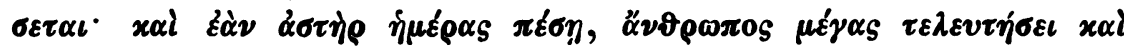

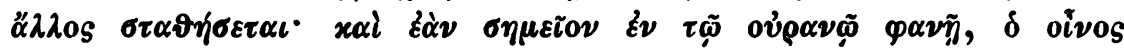

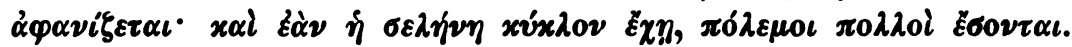

$20 \quad \bar{\gamma} . \Delta \varepsilon x \varepsilon \mu \beta \rho i \omega$ ह่à

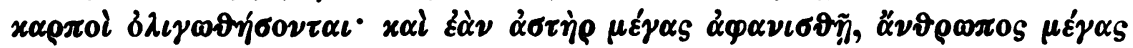

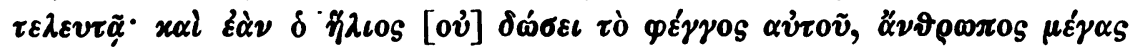

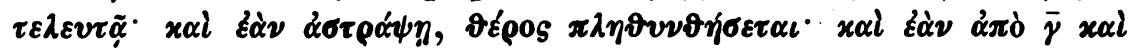

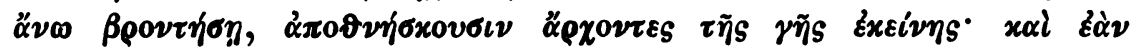

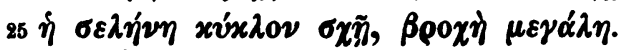

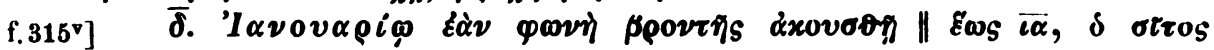

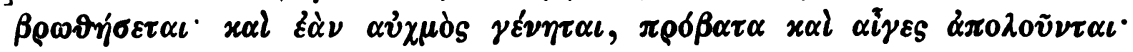

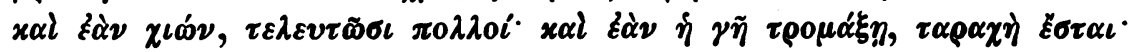

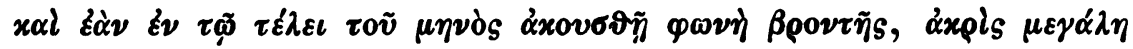

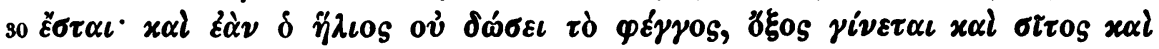

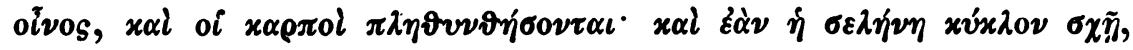

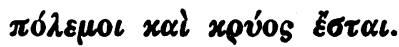

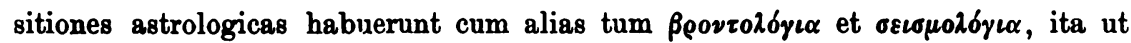
ne imperatores quidem aspernarentur talis conponere, velut imperatoris Heraclii

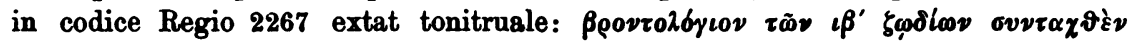

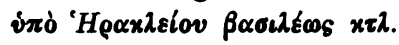

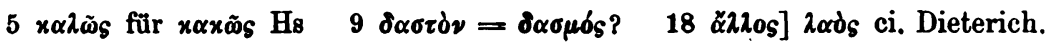




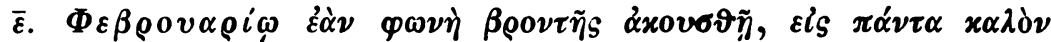

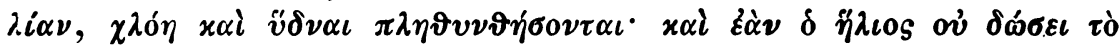

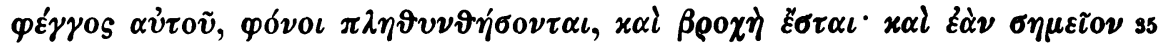

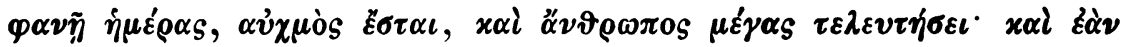

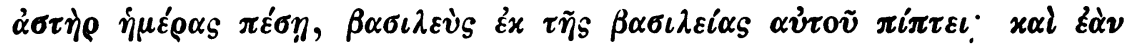

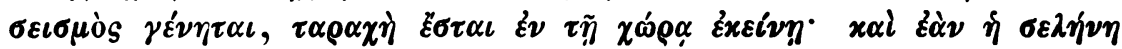

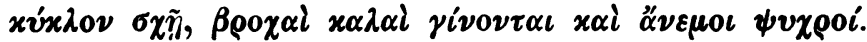

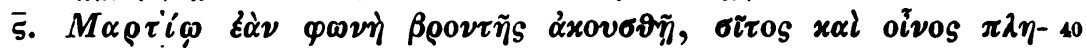

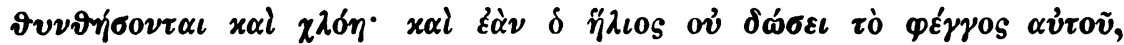

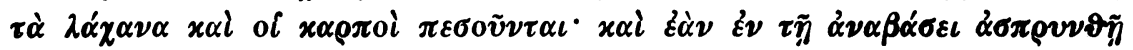

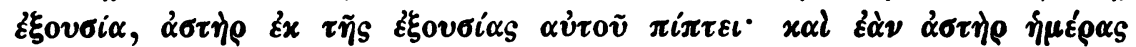

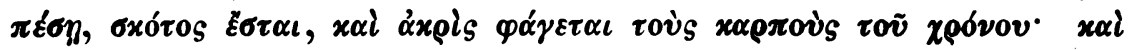

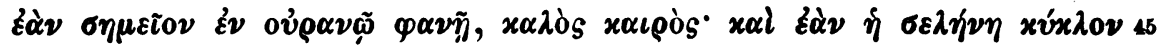

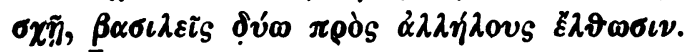

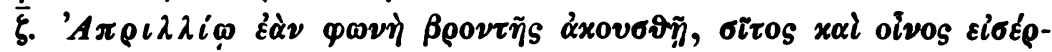

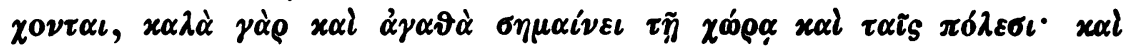

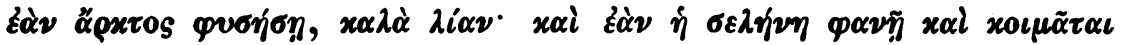

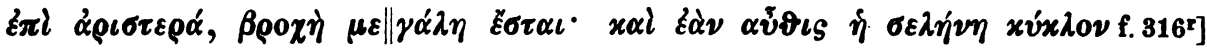

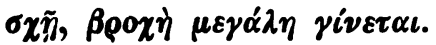

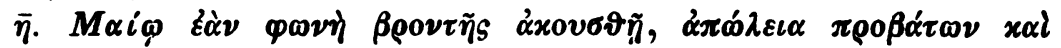

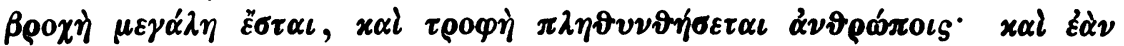

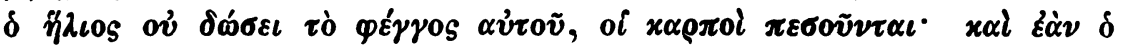

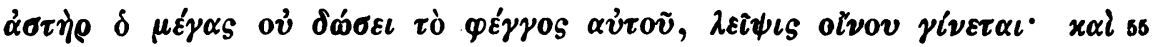

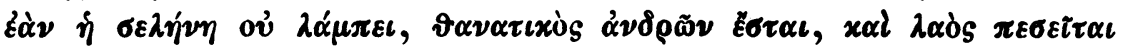

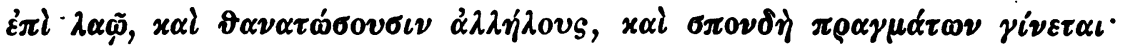

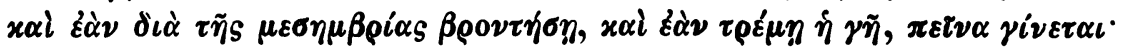

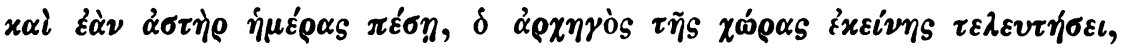

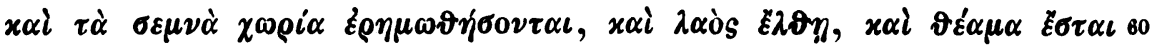

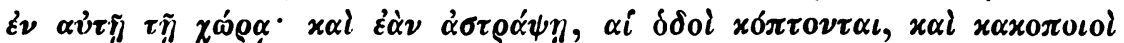

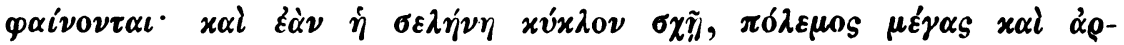

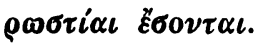

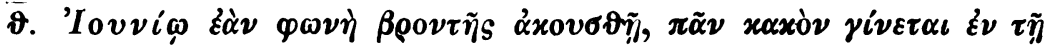

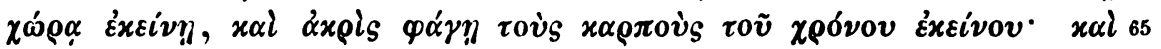

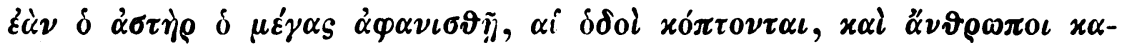

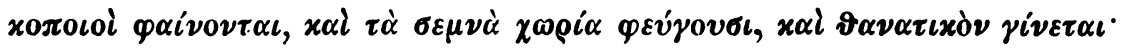

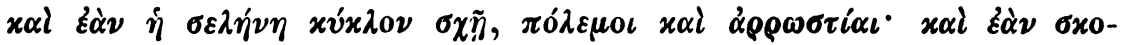

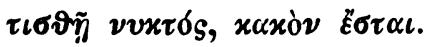

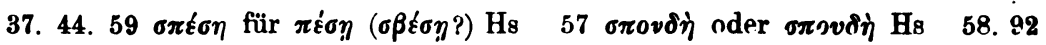

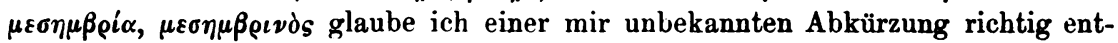
nommen zu haben.

Byzant. Zeitschrift $v 3$ u. 4. 


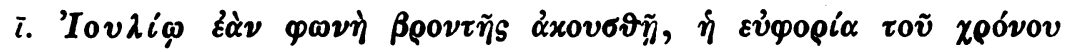

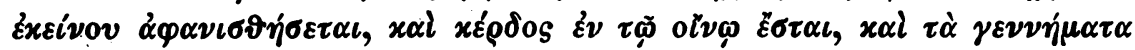

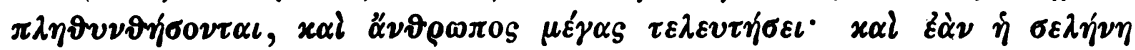

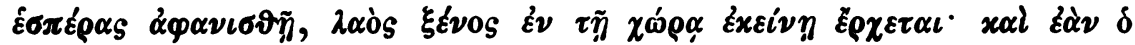

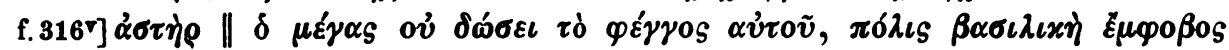

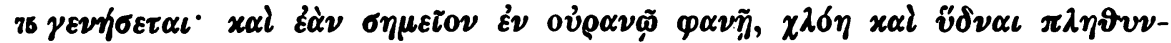

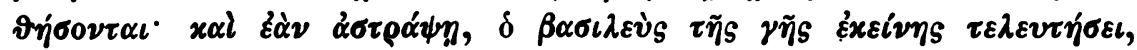

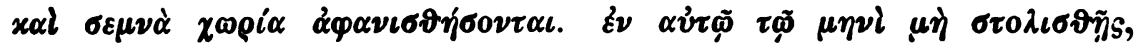

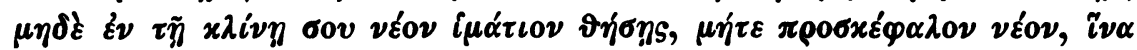

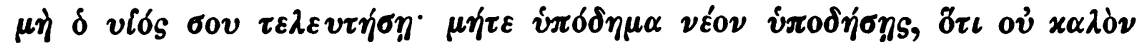

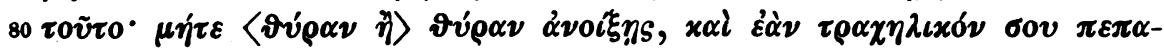

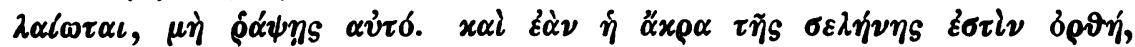

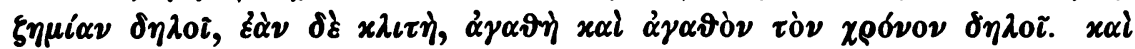

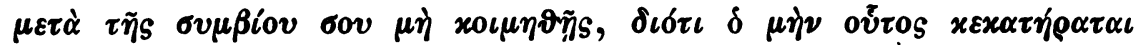

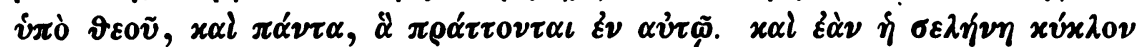

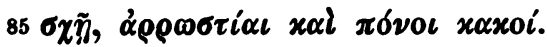

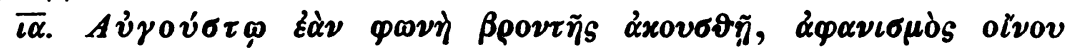

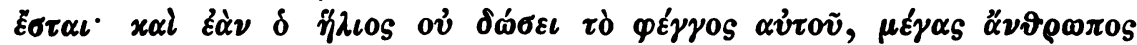

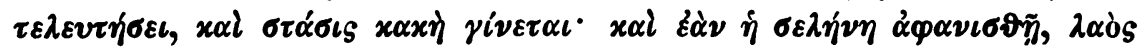

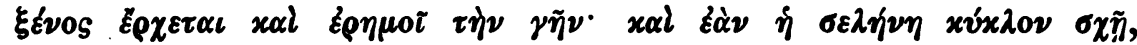

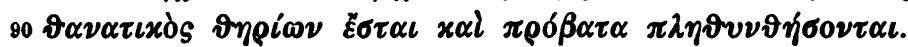

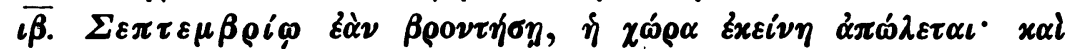

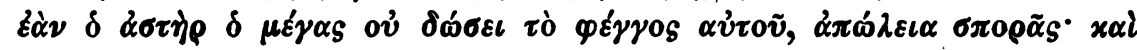

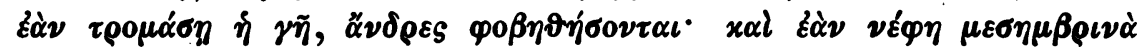

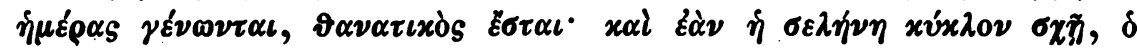

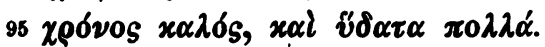

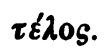

Die Sprache des Traktats, die streng beibehalten worden ist, verrät namentlich durch den ungenauen Gebrauch der Tempora und Modi (Konjunktiv für Futurum!) seine späte Abfassung. Quelle war ein älteres Brontologium, das im Fortgange immer mehr zurückgedrängt wurde durch ein anderes Schriftchen, das aus allen Himmelszeichen schlechthin prophezeite. 'Zu den Lebensregeln über den Juli, den Monat,

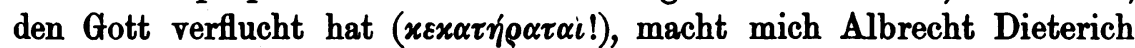
darauf aufmerksam, dafs der Aufgang des Sirius und der Beginn der Hundstage immer als verderblich gegolten haben, wie denn heute noch in Kärnten der Juli für den Trauungen ungünstig erachtet wird (Wuttke, Volksaberglauben p. 23); im Altertum hat man seinen Aufgang beobachtet, um daraus die Beschaffenheit des ganzen Jahres zu

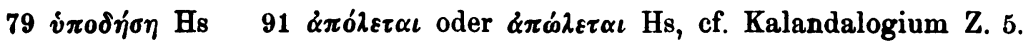


prophezeien, was zu unserer Stelle sehr gut pafst; Cic. de div. I 57: etenim Ceos accepimus ortum caniculae diligenter quotannis solere servare, coniecturamque capere, ut scribit Ponticus Heraclides, salubrisne an pestilens annus futurus sit. nam si obscurior et quasi caliginosa stella extiterit, pingue et concretum esse caelum, ut eius adspiratio gravis et pestilens futura sit: sin illustris et perlucida stella apparuerit, significari caelum esse tenue purumque et propterea salubre.

Gewöhnlich finden sich die Brontologien verbunden mit Kalandalogien, d. h. Schriftchen über die Bedeutung des ersten Januar, je nach seinem Zusammentreffen mit den einzelnen Wochentagen. Auch diese sind meist aus späterer Zeit und anonym überliefert - nur in zwei Parisini (2286 f. $110^{\text {r }}, 3028$ f. $163^{\text {}}$ ) figuriert $\delta \pi \rho \circ \varphi \eta^{\prime} \eta{ }^{N} E \sigma \delta \rho \alpha$ als Verfasser. Ich gebe als Beispiel das Kalandalogium des Vatic. gr. 1823 (misc. s. XV, XVI) f. $103^{\mathrm{v}}$ :

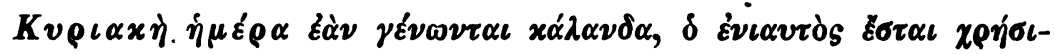

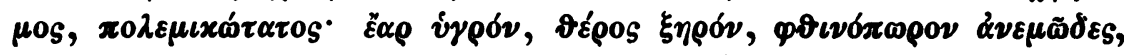

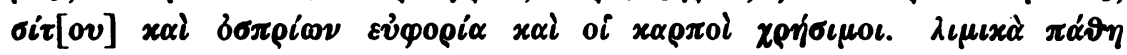

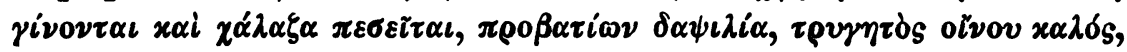

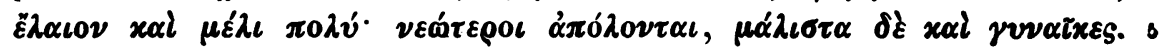
$\tilde{\alpha} \lambda \alpha \tau 0 \mathrm{~s} \lambda \varepsilon \tilde{i} \psi \iota$.

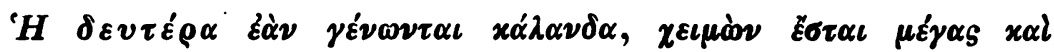

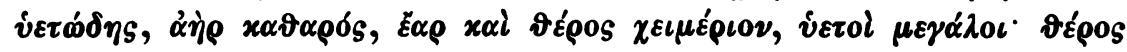

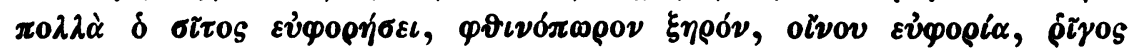

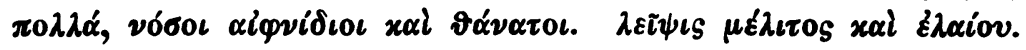

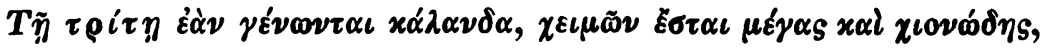

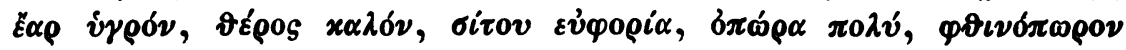

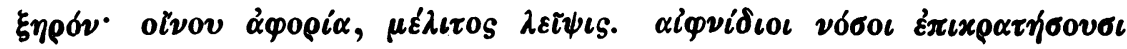

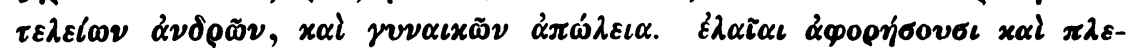

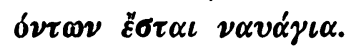

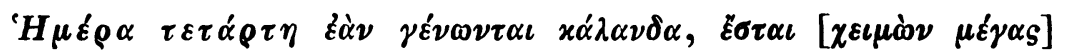

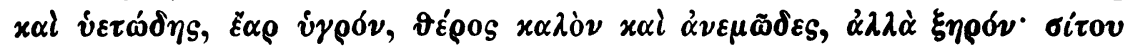

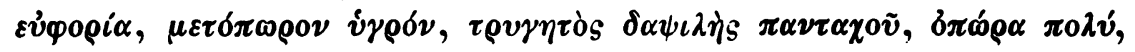

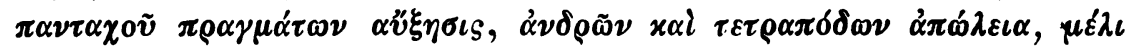

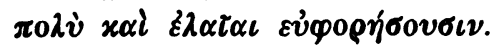

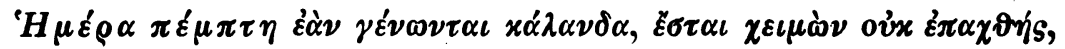
$\varepsilon \alpha \rho \dot{\alpha} \nu \varepsilon \mu \tilde{\omega} \delta \varepsilon S, \vartheta \varepsilon^{\prime} \rho \sigma_{S} x \alpha \lambda \delta \delta$,

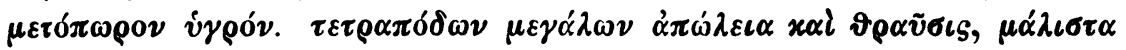

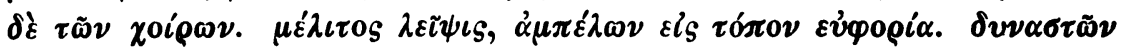

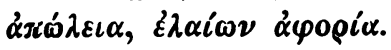

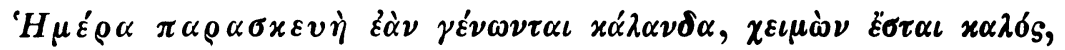




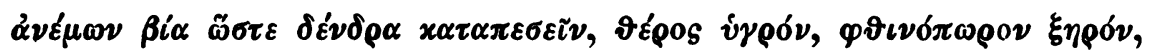

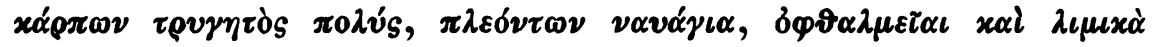

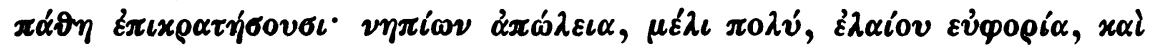

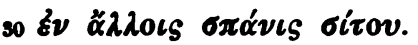

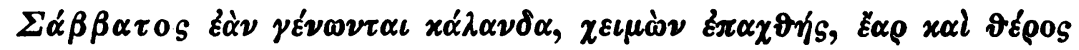

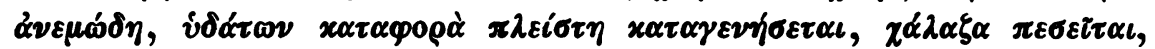

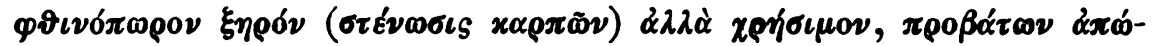

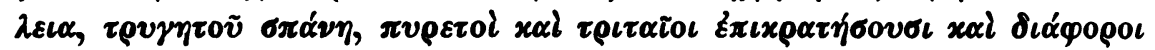

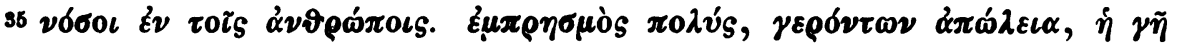

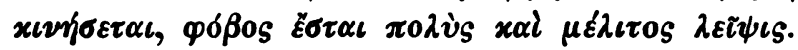

In diesem Kalandalogium ist die Sprachverwilderung noch etwas weiter gegangen als in jenem Brontologium; aufserdem spricht die Durchführung der modernen Wochennamen für eine junge Zeit. Und doch können wir auch hier wieder an Lydus anknüpfen und durch ihn nachweisen, dafs der Aberglaube, der uns hier fast am Ende des Mittelalters entgegentritt, mit seinen Wurzeln bis an dessen Anfang hinabreicht: denn einen Traktat, der dem unsrigen sehr ähnlich war, mufs Lydus als Quelle für de mens. IV 10, wo er diese Anschauung sogar für römisch erklärt, gehabt haben. Des Vergleiches halber setze ich die Stelle ganz hierher:

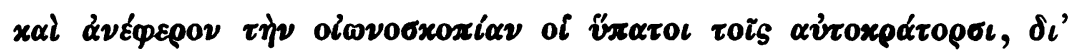

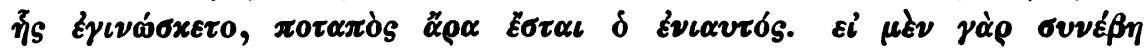

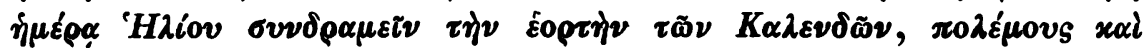

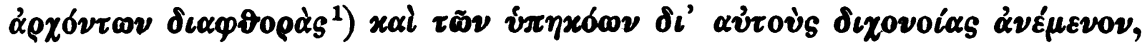

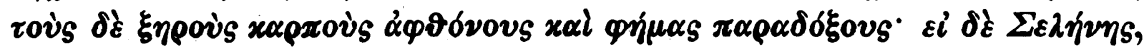

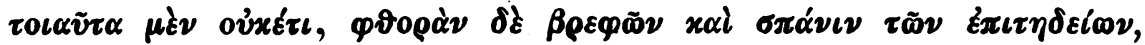

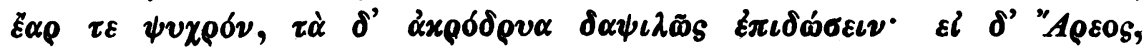

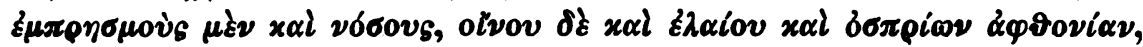

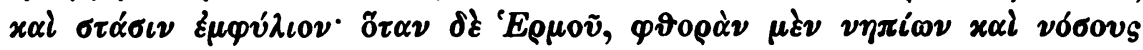

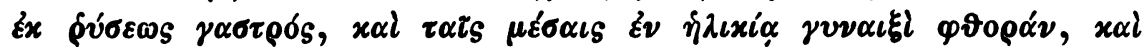

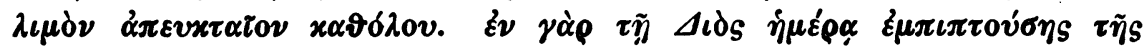

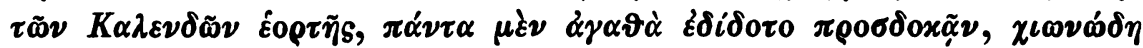

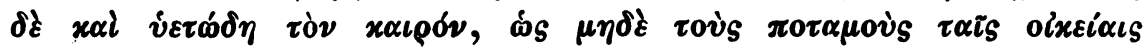

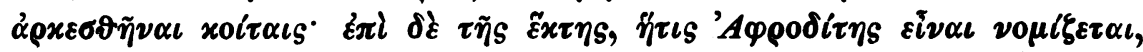

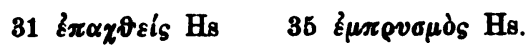

1) In einem von Herrn Geh. Rat Usener mir gütigst zur Verfügung gestellten

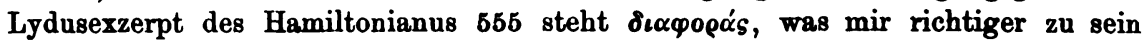
scheint. 


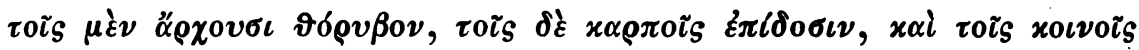

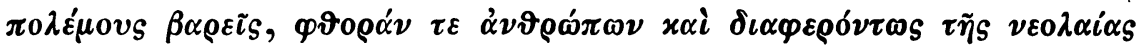

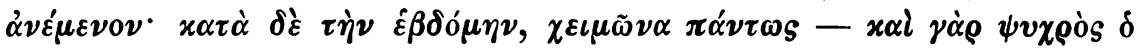

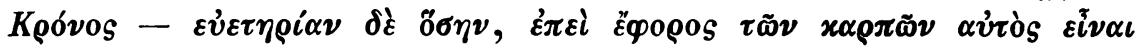

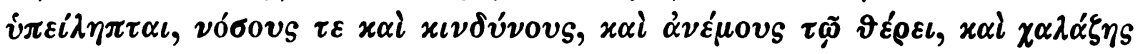

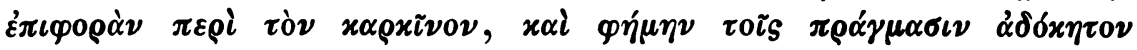
oiฒvíovขo.

Wetzlar.

R. Wünsch. 